\title{
Comparative Analysis of Transport Layer Protocols under Diverse Traffics in Mobile WiMAX Network using NS2 with Improved Scene Generator
}

\author{
Zayed-Us-Salehin \\ Assistant Professor \\ Information and Communication Engineering \\ Department, Noakhali Science and Technology \\ University, Noakhali, Bangladesh
}

\author{
Farjana Afroz Nila \\ Information and Communication Engineering \\ Department, Noakhali Science and Technology \\ University, Noakhali, Bangladesh
}

\begin{abstract}
Worldwide Interoperability for Microwave Access (WiMAX) is a Metropolitan Area Network (MAN) wireless technology that provides broadband Internet access to both fixed and mobile users. Due to limited radio propagation range of wireless mobile devices and variable and unpredictable characteristics of wireless communication medium, there are certainly some effects of the speed of mobiles, and the amount of reliability provided by the underlying Transport Layer on the network performance. In this study, simulation of WiMAX networks is performed using Network Simulator 2 (NS2) to investigate the effects of Maximum Velocity and Pause Time of Mobile Stations and a comparative performance analysis of two commonly used Transport Layer Protocols, namely, Transmission Control Protocol (TCP) and User Datagram Protocol (UDP), is also conducted. TCP variant New Reno is used. For more realistic simulation, NS2 scene generation script is modified. Experiments demonstrate that performance of a WiMAX network is relatively stable when TCP is used. TCP shows overall better performance, especially in case of Packet Delivery Ratio and Average End to End Delay, while throughputs are almost similar for both the protocols, which prefers the use of TCP over UDP in mobile WiMAX networks for all applications.
\end{abstract}

\section{Keywords}

WiMAX, Transport Layer, NS2, TCP, UDP, Scene Generator.

\section{INTRODUCTION}

In recent past, broadband Internet connections were restricted to wireline infrastructure using Digital Subscriber Line (DSL), T1 or cable-modem based connection [1]. But it was always preferred by the providers and subscribers to have a wireless solution. Institute of Electrical and Electronics Engineers (IEEE) 802.16 standard for Broadband Wireless Access (BWA) and its associated industry consortium, WiMAX forum promise to offer high data rate over large areas to many users where broadband is unavailable [1,2,3]. Development of this standard facilitates low cost equipment, ensure interoperability, and reduce investment risk for operators. After its introduction, IEEE 802.16 working group has developed a number of standards for WiMAX. The initial versions of $802.16 / \mathrm{a} / \mathrm{d}$ focused on fixed subscriber stations, the later versions, 802.16-2005 (16e) and 802.16-REV2, included many new features and functionalities needed to support mobility and enhanced quality of service (QoS) [2]. These standards have made it possible to provide BWA to both mobile and fixed users, which was impossible in case of wireline infrastructure. The 802.16 Working Group is currently focusing on the specification for next-generation systems in the $802.16 \mathrm{~m}$ Task Group [2, 3].

WiMAX network based on the IEEE 802.16e, also known as Mobile WiMAX, has gained tremendous momentum in the industrial and academic sectors [4,5]. A great challenge for the Mobile WiMAX providers is to provide the same quality access to both fixed and high speed mobile users, since high speed nodes change their locations frequently and they may require frequent handovers as the probability of crossing the cell area is higher for them. A solution to this may be using a reliable Transport Layer Protocol. Some Transport Layer Protocols, such as User Datagram Protocol (UDP), are not reliable. For getting higher data speed these protocols lose the reliability. On the contrast, connection oriented protocol, such as Transmission Control Protocol (TCP), can provide reliability to the communication by applying some mechanisms such as sliding window, handshaking, acknowledgments, flow control, congestion control etc [6]. Since the introduction of WiMAX was made to provide extreme high speed communication experience, data speed cannot be compromised, but there are some applications where reliable communication is a must. This simulation study investigates and compares the performance of mobile WiMAX network utilizing TCP and UDP as Transport Layer Protocol. Both the protocols are compared using the performance parameters Packet Delivery Ratio (PDR), Throughput, and Average End to End (E2E) Delay.

\section{RELATED WORKS}

A vast amount of research works has been done and are going on to optimize the performance of WiMAX networks. Authors in [7] studied diverse transport layer protocols like TCP, UDP for video streaming over WiMAX. They found UDP has preferable performance than TCP for video streaming. The authors of [8] configured TCP and UDP as an underlying protocol for exchange of data between two wireless mobile nodes in a WiMax access network and had evaluated the effect on performance in terms of end-to-end delay and throughput. The results showed that when handover is triggered the transfer window resets to zero causing higher throughput and end-to-end delay for TCP than UDP. In [9], the performance of IEEE 802.16e was evaluated for UDP and TCP traffic by varying the number of subscriber stations, the distance between the subscriber station and its base station, modulation schemes combined with coding techniques. A comparative study of mixed traffic scenarios for different scheduling algorithms in WiMAX was done in [10]. In this paper, the authors proposed a Greedy-Scheduler that can provide better intra-class protection for TCP flows in 
WiMAX. In paper [11], the performance characteristics of five representative TCP schemes, namely TCP New Reno, Vegas, Veno, Westwood and BIC, in WiMAX networks, under the conditions of correlated wireless errors, asymmetric end-to-end capabilities and link congestion was studied. They proposed a hybrid solution combining the Binary Increase (BI) in congestion avoidance phase, with the Adaptive Decrease (AD) in fast recovery phase, as an ideal candidate in WiMAX networks. Authors in [12] examined performance characteristics of an operational WiMAX testbed upon which they were able to conduct controlled experiments in the absence of competing traffic. They characterized latency, throughput, protocol overhead, and the impact of WiMAX on TCP dynamics. Results showed that scheduling policies and parameter values impact actual performance in ways that are not possible to characterize in generic studies of WiMAX. In [13], the authors analyzed the impact of mobility on the QoS parameters (Throughput, Average Jitter and Average end to end Delay) of a mobile WiMAX network (IEEE 802.16e) with CBR application. Authors of paper [14] examined and compared performance of uplink and downlink bulk data transfer in WiMAX for four TCP variants: New Reno, Cubic, Vegas and Veno. They found that New Reno and Veno provides overall better performance. Analysis of performance characteristics of TCP congestion control algorithms applied by different TCP variants such as TCP Westwood+, Veno and New Reno based on mobile WiMAX is done in [15]. The results of this paper show that TCP Westwood+ and Veno congestion control algorithms are substantially better than New Reno up to $16.5 \%$ and $5.4 \%$ respectively. The authors of [16] evaluated, analyzed and compared the performance of a WiMAX link under different load and traffic conditions. For this purpose they deployed an experimental WiMAX test-bed at the Communication Network Institute (CNI), in University Dortmund.

Studies have been done for evaluating performance of TCP or UDP individually for specific applications in mobile WiMAX network. Comparative study of UDP and TCP has also been done, but for fixed traffic characteristic. This paper evaluates and compares the performance of UDP and TCP by means of extensive simulations under diverse traffics.

\section{SIMULATION ENVIRONMENT}

The result of this study is based on simulations using the network simulator (NS-2) from Lawrence Berkeley National Laboratory (LBNL) in Red Hat 5.0 platform. For the simulation of WiMAX network; a patch "WiMAX Module" from National Institute of Standards and Technology (NIST) is used, which implements the MAC layer (IEEE 802.16) and PHY (OFDMPHY) layer for creating WiMAX environment [17]. As signal propagation model, the "TwoRayGround" Model is used. As QoS specification, only Best Effort (BE) service class is used. Duration of each simulation run is 210 seconds. The traffic starts at 100 second to provide time for initial ranging and other synchronization and authentication. In the simulation area 10 Mobile Stations can move according to the associate scenario. DSDV is used as the routing protocols. Interface Queue (IFQ) of 50 packets is used. The IFQ is a First in First out (FIFO) priority queue where routing packets gets higher priority than data packets. All MAC and Network layer operations of the wireless network interfaces are logged in trace files. Post simulation analyses are performed to each of the trace file by using perl language. Table 1 shows some common parameters used in simulations of this study.
Table 1. Common Simulation Parameters

\begin{tabular}{|c|c|}
\hline Parameter & Selected Value \\
\hline Number of nodes & 10 \\
\hline Base Station Height (m) & 32 \\
\hline Mobile Station Height (m) & 1.5 \\
\hline BS Transmission Power(dB) & $43(20 \mathrm{~W})$ \\
\hline $\begin{array}{c}\text { BS Transmission Range } \\
\text { (meters) }\end{array}$ & 1600 \\
\hline Operating Bandwidth (GHz) & 2.412 \\
\hline $\begin{array}{c}\text { RXThreshold } \\
\text { Packet size (Byte) }\end{array}$ & \begin{tabular}{c} 
TwoRayGround \\
\hline Propagation Model
\end{tabular} \\
\hline Mobility Model & Mobility Model \\
\hline Traffic & TCP/FTP, UDP/CBR \\
\hline
\end{tabular}

\section{MODIFICATIONS MADE TO THE NS2 SCENE GENERATOR}

NS2 uses a Scene Generator program named setdest.cc to create wireless scenario. Using this $\mathrm{C}++$ program, the initial positions of the mobile nodes, the initial movement of the nodes, destination and after reaching the destination, new destination with new movement speed can be designed. To create these positions and movements, this program uses a random number generator header file named rng.h, which returns random number through the function uniform_double() to the function uniform(). The problem with this Scene Generator is that it generates a completely different scenario for each simulation run. Every time it calls the random number generator function uniform(), a unique random number is generated. The set of random numbers are completely different from run to run. As a result, running the program with same parameters does not generate the same scenario; Hence, the sources, destinations, and movements of the nodes are also different. With these types of generated scenes, it is quite impractical to evaluate the effects of node velocity and pause time on the protocols performance. At least the initial and final position should be fixed while varying the velocity and pause time. To accomplish this, before running simulation, some random numbers were generated and saved them on an array. During simulation, whenever random numbers were required to set the initial positions and destinations of the nodes, the numbers from those saved numbered set have been used. But for movement related calls, the original function uniform() has been used. In addition, to put some level of randomness, nodes after reaching to their pause times, when a new destination is set, the uniform() function is used instead of the saved numbers.

\section{PERFORMANCE METRICS}

To evaluate and compare the performance of the Transport Layer Protocols, three different quantitative metrics have been used. They are:

1) Packet Delivery Ratio (PDR): PDR is the ratio of data packets delivered to the destination to those generated by the sources and is calculated as follows [18]: 
PDR $=$ (Number of Packets Received/Number of Packets sent) x 100

2) Throughput: Throughput is the number of bytes received successfully and is calculated by [18]:

Throughput $=($ Number of Packets Received $\times 8$ /Simulation Time $* 1000000) \quad$ Mbps

3) Average End to End (E2E) Delay: Average End-to-End delay is the average time of the data packet to be successfully transmitted across a MANET from source to destination. It includes all possible delays such as buffering during the route discovery latency, queuing at the interface queue, retransmission delay at the MAC (Medium Access Control), the propagation and the transfer time, processing time at Transport Layer [18]. The average E2E delay is computed by,

$\mathrm{D}=\frac{\sum_{i=1}^{n} R i-S i}{n} \mathrm{~m} \mathrm{sec}$

Where D is the average End-to-End delay, $n$ is the number of data packets successfully transmitted over the MANET, ' $\mathrm{i}$ ' is the unique packet identifier, $R_{i}$ is the time at which a packet with unique identifier ' $\mathrm{i}$ ' is received and $S_{i}$ is the time at which a packet with unique identifier ' $i$ ' is sent. The Average End-to-End Delay should be less for high performance.

\section{SIMULATION RESULTS AND DISCUSSION}

Simulations have been run to evaluate the effects of node velocity and pause time on the protocols for the comparison purpose; and hence the simulation results and their analysis are subdivided into two sections. Firstly, the results found by varying maximum velocities of the nodes are analyzed, and then results obtained by varying pause times of mobile nodes are investigated.

\subsection{Result Analysis: Varying the Maximum Node Velocity}

The modified Scene Generator that has been used is able to make the initial position and destination of a mobile node fixed, but when the velocities of nodes are varied; they vary randomly as the modified generator has not been used for defining the movement velocities of nodes. For example, if a node moves from position A to B with a speed $9 \mathrm{~m} / \mathrm{s}$ where maximum speed is set to $10 \mathrm{~m} / \mathrm{s}$, it is possible that for maximum speed $20 \mathrm{~m} / \mathrm{s}$, the same nodes moves from A to B with a speed that is $8 \mathrm{~m} / \mathrm{s}$. It is obvious that the speed of the nodes have a significant impact on the network performance, therefore, this impact cannot maintain a sequence in this simulation. But still measuring the stability of the protocols using these scenarios by investigating how the protocols behave with speed variations of the nodes is possible. For this purpose, the Maximum Node Speed is varied from 2 meters/second to 20 meters/second with an interval of 2 units. In all cases, Pause Time is set to 0 second.

\subsubsection{Effects of the Node Velocity on Performance Metrics}

The PDR curves in Figure 1 show that, in case of connection oriented TCP, PDR values are always within the 99-100\% range, while UDP produces lower PDR values, and the values vary significantly with varied Maximum Nodes Speed, indicating the instability of the UDP network caused by edge nodes and unreliable nature of UDP.

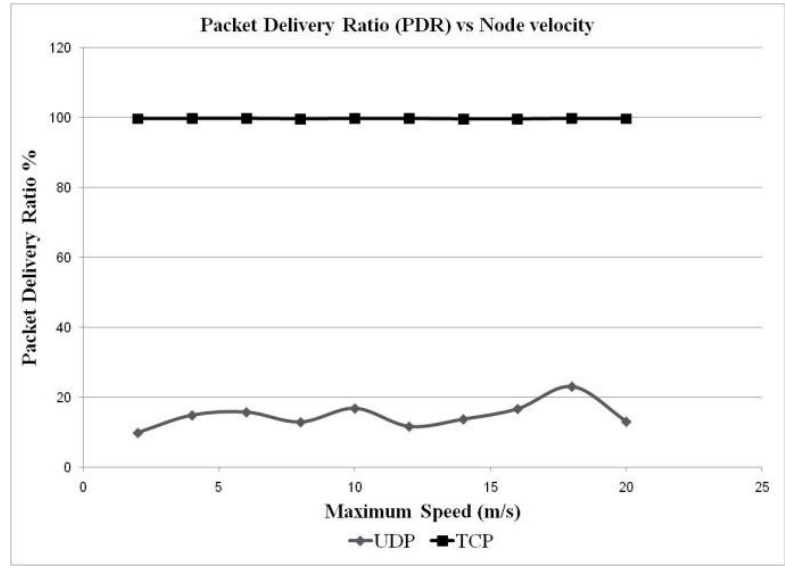

Fig 1. Packet Delivery Ratios versus Node Velocity

The Throughput curves of Figure 2 illustrates that TCP produces better throughputs than UDP. There are always some significant gaps between the two Throughput curves except the point indicating $16 \mathrm{~m} / \mathrm{s}$, where UDP gets slightly better throughput than TCP. Investigation of the scenario of this exceptional case reveals that the maximum number of nodes is within the cell range in this particular scenario. After reaching the initial destinations, most of the nodes move towards the BS, which increases the throughput. As TCP always remain stable, movement of nodes toward BS increases its throughput but not as much as UDP.

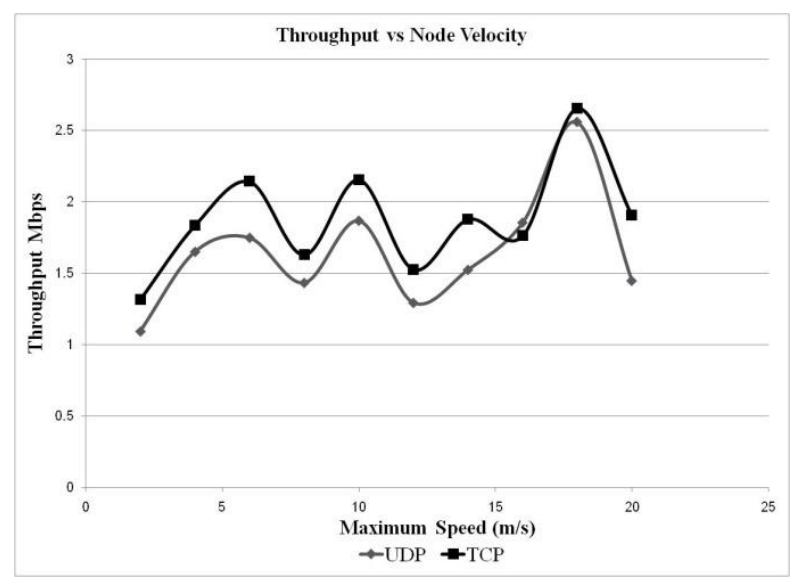

Fig 2. Throughputs versus Node Velocity

Figure 3 shows the E2E delay graphs for the protocols. That proves TCP has relatively stable and lesser E2E Delay values on average. Since number of packets sent in UDP network is higher, they have to wait in queue which increases Average E2E Delay. 


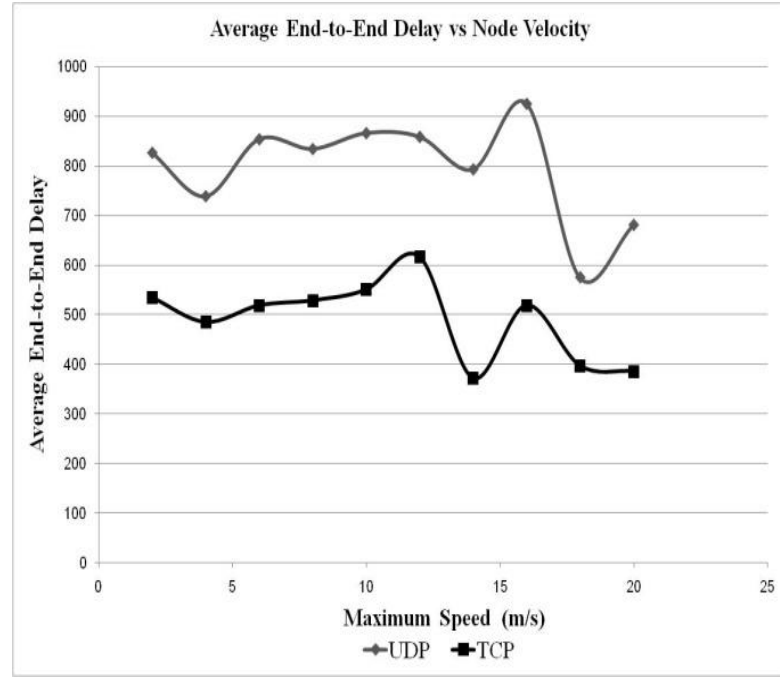

Fig 3. Average End to End versus Node Velocity

\subsection{Result Analysis: Varying the Pause Time}

The Pause Time is varied from 0 seconds to 20 seconds to observe its effects on TCP and UDP. Maximum velocity of nodes is set to $20 \mathrm{~m} / \mathrm{s}$. If within the simulation time, a particular node reaches its destination, then it waits there for the defined Pause Time and after that starts to move for a new destination with a new speed. That means the effect of pause time is initialized only when the nodes reach their destination.

\subsubsection{Effects of Pause Time on the Performance Metrics}

Figure 4(a) shows that UDP PDR values decreases gradually while increasing the pause time. As greater Pause Time means greater possibility of receiving packets since nodes are then not moving, queues get full quickly which results in discarding of packets. Besides, nodes that are paused at the cell edge spend a greater amount of time there. The effects of these edge nodes also decrease the PDR of UDP. In case of $\mathrm{TCP}$, the PDR curve suddenly goes downward for two points indicating the Pause Time 10 and 12 seconds, as Figure 4(b) illustrates. If we consider the range of this variation, it can be ignored and it may be stated that TCP shows a stable nature due to its provided reliability.

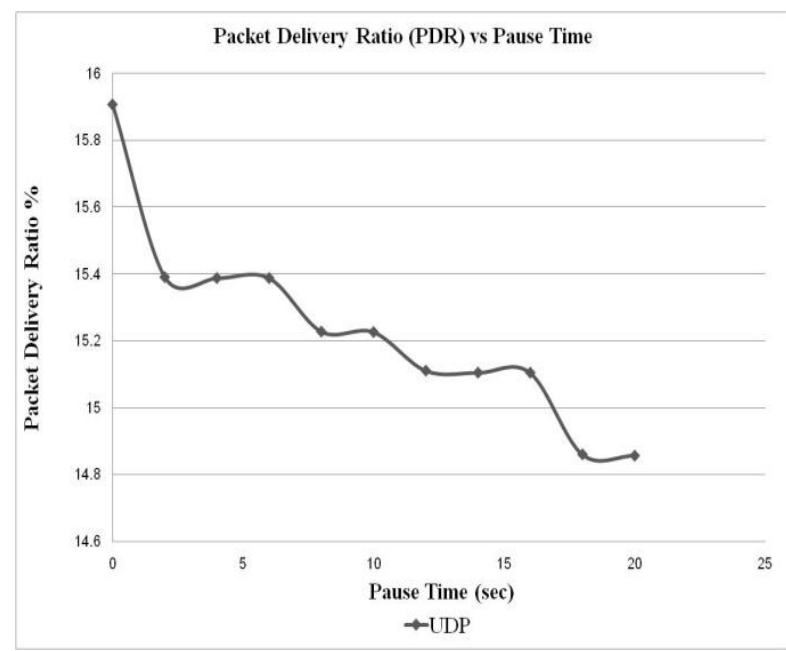

Fig 4(a). UDP Packet Delivery Ratios versus Pause Time

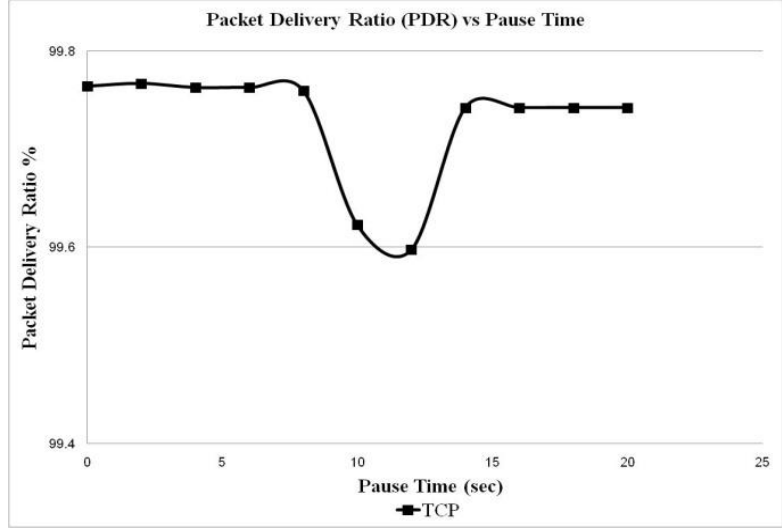

Fig 4(b). TCP Packet Delivery Ratios versus Pause Time

The behavior of the throughput curves for the two Transport Layer Protocols when varying the Pause Times of the Mobile Stations is illustrated in Figure 5.

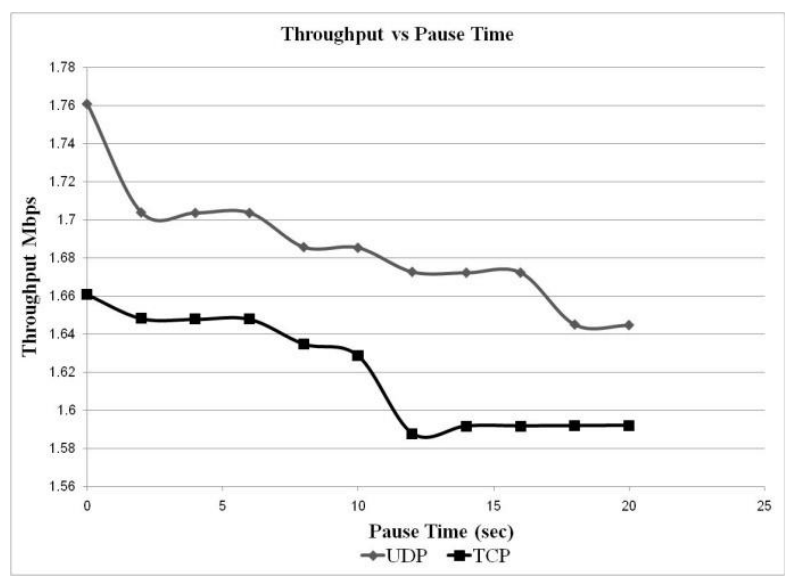

Fig 5. Throughputs versus Pause Time

Both the protocols have lower throughputs at higher Pause Times. The best throughput they show at Pause Time of 0 seconds. The worst case for UDP is found at 20 seconds, and for TCP, throughput drops significantly after 10 seconds and then remains stable afterward. This reduction in throughput is due to the network load that increases with increasing Pause Time. UDP throughput drops mainly for full queues, while TCP suffers from retransmission process for the edge nodes. Lost packets from edge nodes trigger congestion control which reduces throughput for TCP.

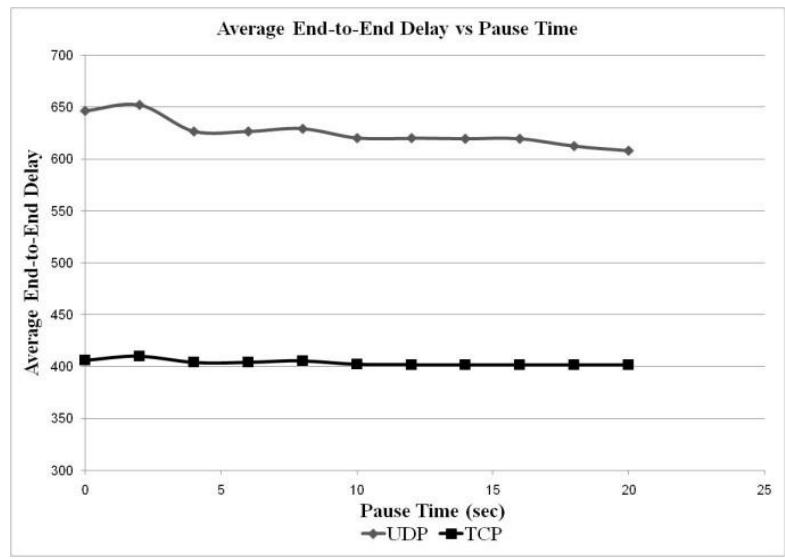

Fig 6. Average End to End Delay versus Pause Time 
The E2E delay curve in Figure 6 illustrates that, UDP suffers from greater delays and delay reduces with increased Pause Time, while TCP curve remains almost flat. Increased Pause Time means some Mobile Stations remains fixed for that amount of time. They may be paused within the cell range or near the cell edge or outside the cell. First two cases are to consider since the nodes that are out of cell range have no effects on the network performance in our simulation. Both the cases that we are considering reduces throughputs for UDP which also reduces E2E delay times due to lesser network loads.

\section{CONCLUSION}

According to the result of simulation, PDR of TCP is much better than UDP, as expected, since TCP is a reliable and connection oriented Transport Layer Protocol. TCP generates, on average, slightly better throughput values, which was unexpected, since TCP passes some extra time to make the network reliable. Simulation results show that the huge numbers of data packets that are sent in UDP network make the receiver queues exhausted and as a result the throughputs are dropped and become almost similar to TCP network Throughputs. When the E2E delays of the protocols are considered, TCP has much less delay than UDP. Stability of a network is an important factor of concern. Simulation illustrates that TCP has much better stability under diverse traffics in comparison of UDP.

\section{REFERENCES}

[1] F. Anwar, M. S. Azad, M. A. Rahman, and M. M. Uddin, "Performance Analysis of Wireless Routing Protocols in Mobile WiMAX Environment", IaENG International Journal of Computer Science, 35(3), August, 2008.

[2] E. Kamran, "Overview of mobile WiMAX technology and evolution", IEEE Communications Magazine, pp. 31- 40, October, 2008.

[3] J. Rakesh, V. Wankhede, and U. Dalal, "A Survey of Mobile WiMAX IEEE 802.16m Standard", International Journal of Computer Science and Information Security, vol. 8, no. 1, pp. 125- 131, April, 2010.

[4] S. More and D. K. Mishra, "4G Revolution: WiMAX Technology", IEEE 978-1-4673-2590-5/12, 2012.

[5] L. Sarperi, M. Hunukumbure, and S. Vadgama, "Simulation Study of Fractional Frequency Reuse in WiMAX Networks" FUJITSU Sd. Tech. J., 44(3), pp. 318- 324, July, 2008.

[6] L. Parziale, D. T. Britt, C. Davis, F. Forrester, W. Liu, et al., "TCP/IP tutorial and technical Overview", International Technical Support Organization (IBM Redbooks), pp. 201-228, 2001.

[7] G. Kaur, N. Kaur and P. S. Tiwana, "Survey of UDP, TCP for video streaming in WiMAX", International Journal of Modern Computer Science and Applications, vol. 4, issue 3, pp. 7-9, May, 2016.

[8] K. D. Mayoof, R. Hassan, A. S. Ahmed and A. Marwan, "Performance Evaluation of Handover in WiMax with TCP and UDP as Underlying Protocol", Journal of Computer Science, pp. 936-942, 2015.

[9] A. N. Naqvi, A. M. Abbas and T. A. Chouhan, "A Performance Evaluation of IEEE 802.16e Networks for TCP And UDP Traffics", International Journal of Engineering Research \& Technology, vol. 1, issue 8, pp. 1-8, October, 2012

[10] M. Alizadeh, R. A. Dziyauddin, D. Kaleshi and A. Doufexi, "A comparative study of mixed traffic scenarios for different scheduling algorithms in WiMAX", In Proceedings of IEEE $75^{\text {th }}$ Vehicular Technology Conference, 2012.

[11] K. Tsiknas and G. Stamatelos, "Performance Evaluation of TCP in IEEE 802.16 Networks", In Proceedings of IEEE Wireless Communications and Networking Conference, pp. 2951-2955, 2012.

[12] J. M. Westall and J. J. Martin, "Performance Characteristics of an Operational WiMAX Network", IEEE Transactions on Mobile Computing, vol. 10, no. 7 , pp. 941-953, July, 2011.

[13] K. Bala and K. Ahuja, "Impact of Mobility on QoS of Mobile WiMax Network With CBR Application.", International Journal of Advancements in Technology, vol. 2, no 3, pp. 423-429, July, 2011.

[14] E. Halepovic, Q. Wu, C. Williamson and M. Ghaderi, "TCP over WiMAX: A Measurement Study", In Proceedings of IEEE International Symposium on Modeling, Analysis and Simulation of Computers and Telecommunication Systems, 2008.

[15] T. W. Kim, S. H. Chung, I. S. Yoon and J. S. Kim, "Effects of Handover on TCP Congestion Control Algorithms over Mobile WiMAX", In Proceedings of 5th IEEE Consumer Communications and Networking Conference, pp. 1230-1231, 2008.

[16] F. Z. Yousaf, K. Daniel and C. Wietfeld, "Performance Evaluation of IEEE 802.16 WiMAX Link with Respect to Higher Layer Protocols", IEEE International Symposium on Wireless Communication Systems, Trondheim, 2007.

[17] The Network Simulator NS-2 NIST add-on, IEEE 802.16 model (MAC+PHY), National Institute of Standards and Technology- Draft 1.2.1, 2009.

[18] P. Periyasamy and E. Karthikeyan, "Impact of Variation in Pause Time and Network Load in AODV and AOMDV Protocols", International Journal of Information Technology and Computer Science, vol. 3, pp. 38-44, April, 2012. 